\title{
Clinical practice guideline on emergency management of acute apical periodontitis (AAP) in adults
}

This guideline has been developed by the Canadian Collaboration on Clinical Practice Guidelines in Dentistry (CCCD) and was approved by the Council of the CCCD on 6 December 2002. The full version of the guideline is available online at www.cccd.ca, along with the methods used in its development. Clinical practice guidelines are systematically developed statements to assist practitioner and patient decisions about appropriate oral healthcare for specific clinical circumstances. They should be used as an adjunct to sound clinical decision-making. CCCD guidelines are updated on a regular basis as new research information becomes available.

The dentists' quick reference format as available on the CCCD website or in pdf format on the site is shown below.

Goal This guideline is intended to aid clinicians in ensuring pain relief for people who have acute apical periodontitis (AAP).

Definition AAP is a periapical inflammation resulting from an untreated, non-vital pulp.

\section{Features of AAP}

- Marked pain to biting or percussion;

- Pain is constant and has occurred over a short time;

- Rarely sensitive to thermal changes;

- Delayed or no response to vitality tests;

- No radiographic changes;

- There may be widened periodontal ligament space.

Exclusions The recommendations in this guideline do not apply to patients with swelling, periapical radiolucency nor systemic changes.

\section{Recommendations}

- Non-surgical endodontic therapy (root canal) should be started on the affected tooth as soon as possible.

- Patients should be given the appropriate dose of analgesics [non-steroidal anti-inflammatory drugs (NSAID) if not contra-indicated] pre-operatively, or immediately post-operatively. This should be continued as needed to control pain.

- In the event that endodontic therapy cannot be started immediately, appropriate analgesics should be prescribed.

- For some people, extraction may be an alternative to endodontic therapy.

- If the tooth is in hyperocclusion, the occlusion should be relieved if possible.

- Antibiotic therapy is not indicated for this condition, nor is bony trephination. 


\section{Clinical practice guideline on treatment of acute apical abscess (AAA) in adults}

This guideline has been developed by the Canadian Collaboration on Clinical Practice Guidelines in Dentistry (CCCD) and has been approved by the Council of the CCCD. The full version of the guideline will be available online at www.cccd.ca, along with the methods used in its development. Clinical practice guidelines are systematically developed statements to assist practitioner and patient decisions about appropriate oral healthcare for specific clinical circumstances. They should be used as an adjunct to sound clinical decision-making. CCCD guidelines are updated on a regular basis as new research information becomes available.

The dentists' quick reference format as available on the CCCD website or in pdf format on the site is shown below.

Goal This guideline is intended to aid clinicians in ensuring pain relief for people who have acute apical abscesses (AAA).

Definition AAA is a periapical inflammation resulting from an untreated, non-vital pulp.

\section{Features of acute abscess}

- Tooth is non-vital

- Pain

- Rapid onset

- From slight tenderness to intense, throbbing pain

- Marked pain to biting or percussion

- Swelling

- Palpable, fluctuant

- May be a localised sense of fullness

- Radiographic changes

- No changes to large periapical radiolucency

\section{Recommendations}

- In the case of localised and diffuse abscesses, drainage should be started as soon as possible. This may include non-surgical endodontic treatment (root canal therapy), incision and drainage, or extraction, depending upon the clinician's judgement and taking into account the patient's preferences.

- If immediate drainage is not possible, appropriate analgesia (NSAID) should be recommended until the infection can be drained adequately.

- Systemic antibiotics provide no additional benefit over drainage of the abscess in the case of localised infections.

- In the presence of systemic complications (fever, lymphadenopathy, cellulitis), diffuse swelling or a patient with medical indications, antibiotics may be a helpful addition. There is no evidence to recommend one antibiotic over another in the management of AAA with systemic complications.

- Antibiotic therapy may be indicated when drainage cannot be achieved.

- Patients should be given the appropriate dose of analgesics (NSAID if not contra-indicated) pre-operatively, and/or immediately postoperatively. This should be continued as needed to control pain.

- Antibiotic therapy is not indicated in otherwise healthy patients nor when the abscess is localised. 


\section{The CCCD guidelines}

\section{Commentary \\ by Anne-Marie Glenny}

Evidence-based practice encourages the practitioner to look for and make sense of the available research evidence in order to apply it to everyday clinical problems. It should inform, not replace, clinical judgement and experience by integrating the best evidence with clinical expertise and patient preferences. This implementation of research findings into clinical practice is a challenging task.

Clinical practice guidelines are commonly seen as a tool for helping to close the gap between research evidence and clinical practice. ${ }^{1}$ The role of these "systematically developed statements to assist practitioner and patient decisions about appropriate health care for specific clinical circumstances" ${ }^{2}$ is not to limit or replace clinical judgment but to provide a comprehensive, critical summary of the research findings in the form of easily implemented recommendations for clinical care. ${ }^{3}$

Over the last decade there has been much interest in the development of guidelines, although the rigour of the development process and the quality of existing guidelines varies considerably. ${ }^{4}$ The development of practice guidelines is a complex task that requires many skills, including literature searching, appraisal of scientific evidence, expertise in group decision-making and the presentation of complex information in comprehensible forms. ${ }^{5}$ If guidelines are developed without the proper skills and resources, can they truly assist patients and practitioners in making the most appropriate decisions about healthcare? In order to improve patient care, should not clinical practice guidelines have to meet minimum quality criteria?

An international group of researchers and policy-makers (the Appraisal of Guidelines Research and Evaluation (AGREE) collaboration; see www.agreecollaboration.org) is concerned with improving the quality and effectiveness of clinical practice guidelines. The AGREE collaboration have developed an instrument for assessing the key elements of guideline quality, with quality defined as, "confidence that the potential biases of guideline development have been addressed adequately and that the recommendations are both internally and externally valid, and are feasible for practice." The AGREE instrument consists of 23 key items that cover six domains (see below). Each item is scored from 1 (strongly disagree) to 4 (strongly agree). The instrument aims to assist policy-makers and healthcare providers decide which guidelines should be recommended for use in practice. It can also be used as a selfassessment tool for those involved in the process of developing or updating clinical guidelines.

\section{The six domains covered by the AGREE instrument}

1. Scope and purpose of the guideline

2. Stakeholder involvement

3. Rigour of development

4. Clarity and presentation

5. Applicability

6. Editorial independence

Currently there are many national and international organisations with the remit of guideline development. Within dentistry, one such is CCCD (see www.cccd.ca), established in 1999. Although it is involved in research to assess the best methods for disseminating, implementing and evaluating guidelines, its primary role is the development of clinical practice guidelines for the dental profes- sion. The CCCD aims to produce "credible and useful" guidelines by using a development process that is both evidence-based and transparent. The first clinical practice guidelines undertaken by the CCCD, completed in 2002, provided recommendations on emergency management of AAP in adults. Subsequently, a clinical practice guideline on the emergency management of AAA has been completed. Summaries of both of these guidelines are presented (p. 7).

This article considers the quality of the CCCD guidelines in light of the AGREE instrument criteria.

\section{Scope and purpose of the guidelines}

Clinical practice guidelines should present clearly the overall aim of the guideline and the clinical questions covered. For both AAA and AAP guidelines, the aims are clear and the rationale for the choice of topic is provided (issues such as prevalence of the condition, burden of illness, variation in practice and cost of managing the condition). The clinical questions addressed were developed through consensus.

For the AAP guidelines the clinical question addressed was, "In adult patients presenting with acute apical periodontitis resulting from a non-vital pulp, what is the effect on pain relief of various interventions? These interventions include systemic and local pharmacotherapeutics, local surgical measures, extraction, occlusal adjustment or watchful waiting." The clinical question for the AAA guidelines was identical apart from the patient group under consideration, ie, adults presenting with an AAA. The target population to be covered by both sets of guidelines are clearly described, with an outline of the clinical signs and symptoms characterising each condition.

\section{Stakeholder involvement}

It has been suggested that, in order to increase relevance and uptake, the intended users of guidelines should be involved in the development process. The methods used by the CCCD involve a Methodology Resource Group and a Clinical Advisory Group (CAG). For both sets of guidelines the CAG was multidisciplinary, comprising general dental practitioners and endodontists. There was no discussion, however, of gathering patients' experiences or expectations with regard to the management of either AAA or AAP. The involvement of patients within guideline development can ensure that the key issues of concern to them are considered. These issues may well differ from those raised by the healthcare professionals involved and for this reason some guideline development agencies, such as the Scottish Intercollegiate Guidelines Network (SIGN), ensure patients or their representatives are included in all their guideline development groups. ${ }^{6}$

So that clinicians can quickly determine whether or not a guideline is relevant to them, the target users should be clearly defined. The CCCD guidelines are aimed at general dental practitioners, although this is not explicitly stated. Likewise, to enhance the validity of the guidelines for the target users, it is suggested that the guidelines are piloted prior to publication (see www.agreecollaboration.org). This process can identify skills required in the implementation of the recommendations, potential organisational barriers and, again, patients' views. Although extensive peer review of the CCCD draft guidelines was undertaken, there is no description of the guidelines being piloted by general dental practitioners. 


\section{Rigour of development}

The methods used by the CCCD to identify, appraise and synthesise the relevant research evidence are, in general, rigorous and explicitly stated in both the AAP and the AAA guidelines. As previously mentioned, the clinical questions to be addressed were established through a consensus process. Following this, systematic reviews were undertaken of clinical trials relevant to the clinical questions. The criteria for selecting the trials for the systematic reviews, for each set of guidelines, were clearly defined and the process carried out independently by two reviewers.

For both sets of guidelines, searches of Medline, EMbase and the Cochrane Central Register of Controlled Trials were undertaken from database inception through to August 2001 (for the AAP guidelines) or March 2002 (AAA guidelines). The search strategies used are presented in full.

The systematic reviews undertaken were limited to published articles only, in either the French or English language. In the most recent set of guidelines (AAA) the reviewers recorded how many non-English or non-French language citations were identified so as to gauge the possible impact of limiting the review with regard to language of publication. Eighteen potentially relevant articles were identified but were not reviewed because of lack of resources for translation.

Ideally systematic reviews should include both published and unpublished studies, irrespective of language, as there is good evidence of publication bias according to the results of a study. Studies with statistically significant findings are more likely to be submitted for publication, ${ }^{7}$ published in English-language journals ${ }^{8}$ and be published sooner ${ }^{9}$ than studies with more equivocal results. Resources do not always allow such a comprehensive review of the literature, however, and more recent research suggests that systematic reviews based on a search of English language literature only will often, but not always, produce results similar to those obtained through more comprehensive reviews. ${ }^{10}$ Egger et al suggest that financial and time constraints be considered when deciding the degree of comprehensiveness appropriate for a given review. ${ }^{10}$

The assessment of the quality of the individual studies included in a systematic review and a statement on how biases identified might affect the outcomes are essential in establishing the believability of a review's findings. Within the systematic reviews undertaken as part of the CCCD guideline development process, all studies included were assessed using the Jadad checklist ${ }^{11}$ and the findings of the quality assessment clearly presented. The Jadad checklist is a widely used composite scale that focuses on the internal validity of the trial by examining randomisation, blinding and withdrawals. Each trial is given a score of $0-5$, but it should be noted that scores reflect of the quality of the reporting more than the quality of the trial's methods. For example, a trial can gain points for simply describing the number of dropouts that occurred, irrespective of the number that dropped out, or whether analysis was carried out on an intent-to-treat basis or not. In addition, the scale makes no allowances for the fact that in certain circumstances blinding is not always possible, such as when examining the effectiveness of some surgical procedures. There has been much debate over the use of such composite scales for the assessment of randomised controlled trials (RCT), particularly as recent evidence showed that different scales can give conflicting results. ${ }^{12}$ The assessment of the individual components, without producing an overall summary score, may be preferable.

Evidence tables containing summaries of all included trials $(n=15$ for the AAP guidelines; $n=8$ for the AAA guidelines) were produced and meta-analysis of clinically homogeneous trials was undertaken. The findings of the systematic reviews were reviewed by the relevant CAG and evidence-based recommendations were developed by consensus within the group.
The draft recommendations underwent thorough external review through a survey sent by post to Canadian dentists for whom the guidelines were thought relevant. The survey gathered information on practitioners' views about the need for a clinical practice guideline on the topic, the methods used in the development of the guidelines, the recommendations themselves and their impact. Twenty practitioners provided feedback for the AAP and the AAA guidelines. Based upon this feedback and comments from the CCCD Council the final recommendations (as illustrated in the summary document, p. 8) were produced. Both the draft recommendations and the final recommendations are published in the full document, allowing the reader to establish how the recommendations changed as a result of peer review.

As mentioned previously, evidence tables are given providing summaries of trials included. However, AGREE suggest that there should be an explicit link between the recommendations and the evidence upon which they are based, with each recommendation being linked to the relevant references. The guidelines under review may have benefited from a clearer link between the recommendations and the supporting evidence. The recommendations were, however, graded according to the strength of the supporting evidence (see below).

\section{Grading of evidence used within the CCCD}

- Statements of evidence

- Ia Evidence obtained from meta-analysis of RCT

- Ib Evidence obtained from at least one RCT

- IIa Evidence obtained from at least one well-designed controlled study without randomisation (RCT considered IIa if method of randomisation not clear)

- IIb Evidence obtained from at least one other type of welldesigned quasi-experimental study

- III Evidence obtained from well-designed non-experimental descriptive studies, such as comparative studies, correlation studies and case studies

- IV Evidence obtained from expert committee reports or opinions and/or clinical experiences of respected authorities

- Grades of recommendations

- A Requires at least one RCT as part of a body of literature of overall good quality and consistency addressing the specific recommendation (evidence levels $\mathrm{Ia}, \mathrm{Ib}$ )

- B Requires the availability of well-conducted clinical studies but no RCT on the topic of recommendation (evidence levels IIa, IIb, III), OR small randomised trials with uncertain results (and moderate to high risk of error)

- C Requires evidence obtained from expert committee reports or opinions and/or clinical experiences of respected authorities. Indicates an absence of directly applicable clinical studies of good quality (evidence level IV)

For clinical practice guidelines to be useful they need to incorporate current research. They must not be static documents but there should be a procedure for reviewing and updating the guidelines as appropriate. The CCCD CAG do have formal, standardised processes to ensure the guidelines are kept up-to-date, with new evidence evaluated within 5 years.

\section{Clarity and presentation}

The CCCD promote implementation of the guidelines by producing them in a variety of formats; each guideline is produced as a full report, an executive summary, a quick reference guide and a patient version of the recommendations. The guidelines are clearly 
presented and the recommendations easily identifiable. However, on occasion the recommendations are somewhat vague. For example, "For some patients and in certain situations extraction of the affected tooth is a reasonable alternative."

\section{Applicability}

There are many factors that can prevent a clinical practice guideline from being implemented. Potential organisational barriers and the implication of current resources on the guidelines should be considered. Such factors may come to light during the piloting of a guideline. These issues are not considered within the CCCD guidelines. Similarly, the AGREE collaboration discusses the use of audit for improving the use of a guideline. They recommend that a guideline should clearly define review criteria, derived from the key recommendations in the guideline (www.agreecollaboration.org). No criteria for monitoring and/or audit purposes are presented in the CCCD guidelines.

\section{Editorial independence}

The CCCD primary source of funding is the dentists of Canada, through provincial dental regulatory authorities. The CCCD guidelines are editorially independent from the funding body. The guidelines produced are editorially independent of any funding body and declarations of conflict of interest are stated.

\section{Summary}

For patients and health professionals to benefit from clinical practice guidelines, the guidelines must accurately reflect an unbiased and comprehensive synthesis of the research literature and expert opinion. ${ }^{4}$ Although the guidelines produced by CCCD do not meet all the criteria set out in the AGREE instrument, they are the product of rigorous, scientific methods. On the basis of the guideline development process used, and the transparent reporting, the guidelines are strongly recommended. Despite this, given that the AGREE instrument focuses heavily on the guideline development process, the clinical content of the recommendations and their potential impact on patient outcomes must be considered before adopting guidelines locally.

\section{A general practitioner's view of the guidelines by Terry Simpson}

Both guidelines are well-presented, with information summarised separately for the dental practitioner and patient alike. The majority of practitioners will welcome the aide-memoire for quick reference during a busy day. Alternatively, those wishing to examine the guideline in more detail can read the summary report or the full guideline. The availability of the guideline online will enable access for dentists world-wide.

\section{Acute apical abscess guideline}

The authors identify a serious problem in that $75 \%$ of patients with localised apical abscess are currently prescribed antibiotics. In light of the evidence sourced, this appears to be inappropriate. Although beyond the scope of this guideline, the reasons behind this seemingly high figure are important. Overcoming the barriers to implementation may depend on an investigation and understanding of these reasons. One explanation could be patient demand for antibiotic therapy. This expectation can sometimes put pressure on the practitioner to act against his/her better judgement. The information summary for patients will prove useful in explaining why the dentist chooses drainage of the infection in preference to antibiotic administration for simple abscess.
The key recommendation relating to recognition is that it may be necessary to reschedule drainage because of patient/clinician factors and that, in these instances, analgesia and not antibiotic therapy should be initiated. This may prove the most difficult recommendation for which to achieve widespread acceptance in practice. The results from the dissemination of the guideline to volunteer practitioners (in the guideline's Appendix 3) do, however, contradict this. It is slightly disappointing that the evidence was not available to recommend one antibiotic in preference to another for more widespread infection but the recommendations overall appear to be appropriate, thorough and very relevant to general practice.

\section{Acute apical periodontitis guideline}

This guideline will prove very useful in practice because of the definition of the diagnostic criteria. These will aid the practitioner in distinguishing between reversible pulpitis on the one hand and AAP, as well as between AAP and AAA on the other. The dissemination of the guideline to 20 volunteer practitioners and the subsequent feedback (with approximately $90 \%$ of respondents agreeing on the need for it and $68.4 \%$ agreeing with the conclusions) has assisted in validating the guideline (indeed, similar results were found with the guideline for AAA). Furthermore, most practitioners saw few problems in the recommendations being implemented. Again, the recommendations for AAP appear appropriate, thorough and very relevant to general practice.

\section{Anne-Marie Glenny', Terry Simpson ${ }^{2}$ \\ ${ }^{1}$ Cochrane Oral Health Group, MANDEC, University Dental Hospital of Manchester, Manchester, UK ${ }^{2}$ General Dental Practitioner, Edinburgh, Scotland}

1. Woolf SH, Grol R, Hutchinson A, Eccles M, Grimshaw J. Clinical guidelines: potential benefits, limitations, and harms of clinical guidelines. Br Med J 1999; 318:527-530

2. Lohr K, Field M. A provisional instrument for assessing clinical practice guidelines In Guidelines for Clinical Practice From Development to Use Edited by Field M, Lohr K. Washington DC: National Academy Press; 1992.

3. Shiffman R, Shekelle PG, Overhage M, Slutsky J, Grimshaw J, Deshpande A. Standardized reporting of clinical practice guidelines: a proposal from the conference on guideline standardization. Ann Intern Med 2003; 139:493-498.

4. Hasenfeld R, Shekelle P. Is the methodological quality of guidelines declining in the US? Comparison of the quality of US Agency for Health Care Policy and Research (AHCPR) guidelines with those published subsequently. Quality Safety Health Care 2003; 12:428-434.

5. Shekelle PG, Woolf SH, Eccles M, Grimshaw J. Clinical guidelines: developing guidelines. Br Med J 1999; 318:593-596.

6. Scottish Intercollegiate Guidelines Network. A Guideline Developers' Handbook. Edinburgh: SIGN; 2002, report SIGN 50.

7. loannidis JP. Effect of the statistical significance of results on the time to completion and publication of randomized efficacy trials. J Am Med Assoc 1998; 279:281-286.

8. Egger M, Zellweger-Zahner T, Schneider M, Junker C, Lengeler C, Antes G. Language bias in randomised controlled trials published in English and German. Lancet 1997; 350:158-162.

9. Stern J, Simes R. Publication bias: evidence of delayed publication in a cohort study of clinical research projects. Br Med J 1997; 315:640-645.

10. Egger $M$, Juni $P$, Bartlett $C$, Holenstein $F$, Sterne I. How important are comprehensive literature searches and the assessment of trial quality in systematic reviews? Empirical study. Health Technol Assess 2003; 7:1-76.

11. Jadad A, Moore R, Carroll DP, et al. Assessing the quality of reports of randomised clinical trials: is blinding necessary? Control Clin Trials 1996; 17:1-12.

12. Juni $P$, Witschi $A$, Bloch $R$, Egger $M$. The hazards of scoring the quality of clinical trials for meta-analysis. J Am Med Assoc 1999; 282:1054-1060.

Evidence-Based Dentistry (2004) 5, 7-11.

doi:10.1038/sj.ebd.6400233 